Research Article

\title{
Chinese Herbal Medicine Combined with Conventional Therapy for Blood Pressure Variability in Hypertension Patients: A Systematic Review of Randomized Controlled Trials
}

\author{
Zhuo Chen, ${ }^{1}$ Liqiong Wang, ${ }^{2}$ Guoyan Yang, ${ }^{2} \mathrm{Hao} \mathrm{Xu}^{3}$ and Jianping Liu ${ }^{2}$ \\ ${ }^{1}$ Graduate School, Beijing University of Chinese Medicine, Beijing 100029, China \\ ${ }^{2}$ Centre for Evidence-Based Chinese Medicine, Beijing University of Chinese Medicine, Beijing 100029, China \\ ${ }^{3}$ Cardiovascular Diseases Center, Xiyuan Hospital, China Academy of Chinese Medical Sciences, Beijing 100091, China
}

Correspondence should be addressed to Hao Xu; xuhaotcm@hotmail.com and Jianping Liu; jianping_l@hotmail.com

Received 21 August 2014; Revised 24 September 2014; Accepted 24 September 2014

Academic Editor: Myeong Soo Lee

Copyright (C) 2015 Zhuo Chen et al. This is an open access article distributed under the Creative Commons Attribution License, which permits unrestricted use, distribution, and reproduction in any medium, provided the original work is properly cited.

\begin{abstract}
Objective. The aim of this systematic review is to evaluate effect of Chinese medicine combined with conventional therapy on blood pressure variability (BPV) in hypertension patients. Methods. All randomized clinical trials (RCTs) comparing Chinese medicine with no intervention or placebo on the basis of conventional therapy were included. Data extraction, analyses, and quality assessment were performed according to the Cochrane standards. Results. We included 13 RCTs and assessed risk of bias for all the trials. Chinese medicine has a significant effect in lowering blood pressure (BP), reducing BPV in the form of standard deviation (SD) or coefficient of variability (CV), improving nighttime BP decreased rate, and reversing abnormal rhythm of BP. Conclusions. Chinese medicine was safe and showed beneficial effects on BPV in hypertension patients. However, more rigorous trials with high quality are warranted to give high level of evidence before recommending Chinese medicine as an alternative or complementary medicine to improve BPV in hypertension patients.
\end{abstract}

\section{Background}

It is estimated that there are nearly one billion people suffering from hypertension worldwide, and the number of patients will increase to 1.5 billion by 2050 [1]. Blood pressure variability (BPV) means the degree of blood pressure (BP) fluctuations in a certain period of time. BPV is regarded as a separate index which is different from the BP reflecting cardiovascular activities. Many studies have confirmed that BPV could impact on hypertensive target organ damage and overall prognosis of patients [1-5]. An important factor to improve the prognosis of hypertensive patients is to reduce the BPV effectively. Clinicians pay close attention to lowering pressure steadily nowadays. There have been many studies that elucidated the relationship between western medicine and BPV. Although the results are inconclusive, most studies have shown that calcium channel blockers (CCB) are the most effective to reduce the BPV, especially amlodipine. Amlodipine in combination with other drugs may be more effective [6-8].

Short-term BPV within the 24-hour period is easier to obtain measured results. Ambulatory blood pressure monitoring $(\mathrm{ABPM})$ is a way to assess the short-term BPV at present. By ABPM, a large amount of cross-sectional studies confirmed the increased BPV indicating the aggravated target organ damage [9]. A quantitative analysis of 155 Chinese people's ABPM results showed that BPV of hypertensive patients was higher than that of healthy people [10].

Western medicine puts emphasis on quickly and effectively lowering $\mathrm{BP}$ and helps patients reach target $\mathrm{BP}$ as soon as possible. But even effective long-term control of BP by western medicine may not fully achieve goals of 
protecting target organs against damage, because reversing the target organ damage is a long process. Chinese medicine highlights the overall concept, self-regulation mechanism, and multitargets action and often plays an important role in the protection of target organ of hypertensive patients [11]. However, whether Chinese medicine is effective on BPV remains unclear. So, we design this systematic review to evaluate effect of Chinese medicine on BPV by ABPM in hypertension patients.

\section{Methods}

2.1. Search Strategy. Two reviewers (Z. Chen and L. Q. Wang) searched the following databases from their inception to April 15, 2014, for the identification of randomized clinical trials (RCTs) assessing Chinese herbal medicine for BPV: The Cochrane Library, Pubmed, China National Knowledge Infrastructure (CNKI), Chinese Scientific Journal Database (VIP), Wanfang Databases, and Sino-Med Database. There was no limitation in languages. We also searched references of included reviews, just in case eligible trials were missed. We searched using the following searching terms: "blood pressure variability," "BPV," "essential hypertension," "standard deviation," "coefficient of variability," "traditional Chinese medicine," "Chinese herbal medicine," and "randomized controlled trial." Chinese pinyin of these terms were "xue ya bian yi," "xue ya bo dong," "xue ya bian yi xing," "xue ya bo dong xing," "zhong yao," "zhong chengyao," "zhong cao yao," "zhong xi yi," "ke li," "zhong yi yao," "zhong yi," and "sui ji." Based on different characteristics of literature databases, we adapted the search strategies appropriately which are shown in Table 1.

\subsection{Inclusion Criteria}

2.2.1. Study Design. RCTs were included regardless of blind method and language.

2.2.2. Participants. Patients with BPV regardless of age and race were included, who were diagnosed by one of the following diagnostic criteria: WHO/ISH Hypertension Prevention Guide (1999 [12]/2003 [13]) or Chinese Hypertension Prevention Guide (2005 [14]/2010 [15]). Patients with severe liver and kidney dysfunction and serious complications were excluded.

2.2.3. Interventions. Chinese herbal medicine was used as intervention in at least one group of the study, form of which can be decoction, granule, and Chinese patent medicine (capsule/tablet). The method of application was restricted to orally taken; therefore, injections were excluded. Control groups were no treatment, placebo, or conventional medicines. Trials of Chinese herbal medicine in combination of conventional medicine compared to conventional medicine alone were also included, if the conventional medicine applied in both groups was the same. Trials with a treatment course of less than four weeks were excluded. Trials with more than one kind of Chinese herbal medicine as intervention were also excluded.
2.2.4. Outcome Measures. Primary outcome measures were BPV (measured by standard deviation (SD) and coefficient of variability $(\mathrm{CV})$, at three different time points: 24 hours, day, and night), BP (24 hours, day, and night), and symptom improvement rate which had uniform criteria, that is, clinical guideline of new drugs for traditional Chinese medicine. Among all these indicators, BPV and ambulatory BP are both indispensable. Secondary outcome measures were adverse events, quality of life (QOL), the abnormal rhythm of BP reverse rate, and nighttime $\mathrm{BP}$ decrease rate.

2.3. Data Extraction and Quality Assessment. Two authors (Z. Chen and L. Q. Wang) performed data extraction (Figure 1) independently according to a predesigned form. Disagreements during cross-checking of the data extraction form were resolved by consensus or consultation from a third author (J. P. Liu). We assessed the methodological quality of these trials by risk of bias tool which was recommended by Cochrane Handbook [16]. Specific items of the risk of bias tool were as follows: selection bias (random sequence generation, allocation concealment), performance bias (blinding of participants and personnel), detection bias (blinding of outcome assessment), attrition bias (incomplete outcome data), reporting bias (selective outcome reporting), and other biases. Each item for all included trials was judged into "high risk," "unclear," and "low risk."

2.4. Data Analysis and Synthesis. Revman 5.2 software provided by the Cochrane Collaboration was used for data analyses. We expressed dichotomous data as risk ratio (RR) and its $95 \%$ confidence intervals (CI) and continuous outcome as mean difference (MD) and its 95\% CI. Since all included trials applied different interventions, we did not pool the data due to the clinical heterogeneity. We performed qualitative description of the data synthesis.

We also failed to conduct a funnel plot to explore publication bias, because the number of included studies was less than nine for each outcome.

\section{Results}

3.1. Description of Included Trials. 474 trials were identified from six databases. Among them, 96 records were removed because of duplicates. By screening titles and abstracts, we excluded 263 records for reasons of animal experiment, traditional review, improper comparison, or nonprimary hypertension. By browsing full-text article, we excluded 102 records for reasons of improper comparison, nonprimary hypertension, complications, uncorrelated outcomes, or duplicate publication. At last, a total of 13 articles [17-29] that met inclusion criteria were included into this systematic review. (Basic characteristics of included studies are presented in Table 2.)

3.1.1. Study Characteristics. The 13 trials were published in Chinese from 2007 to 2013, of which seven [17-20, 27-29] were academic dissertations, five [21-25] were journal articles, and one [26] was conference paper. All trials were carried 
TABLE 1: Search strategy.

(1) Cochrane Central Register of Controlled Trials (CENTRAL) on The Cochrane Library

\#1 MeSH descriptor: [Medicine, Chinese Traditional] explode all trees

\#2 MeSH descriptor: [Drugs, Chinese Herbal] explode all trees

\#3 \#1 or \#2

\#4 MeSH descriptor: [Hypertension] explode all trees

\#5 "standard deviation" or SD

\#6 "coefficient of variability" or CV

\#7 \#5 or \#6

\#8 \#4 and \#7

\#9 "blood pressure variability" or "BPV” or "blood pressure fluctuation"

\#10 "Randomized Controlled Trial" or "RCT"

\#11 \#8 or \#9

$\# 12 \quad \# 3$ and \#10 and \#11

(2) PubMed

\#1 "Hypertension" $[\mathrm{MeSH}]$

\#2 “medicine, Chinese traditional” [MeSH Terms]

\#3 “drugs, Chinese herbal” [MeSH Terms]

\#4 \#2 OR \#3

\#5 “randomized controlled trial" [All Fields] or RCT [All Fields]

\#6 BPV [All Fields] OR "blood pressure variability" [Title/Abstract] OR "blood pressure fluctuation" [Title/Abstract]

\#7 “standard deviation" [Title/Abstract] or SD [All Fields]

\#8 “coefficient of variability" [Title/Abstract] or CV [All Fields]

\#9 \#7 OR \#8

\#10 \#1 AND \#9

\#11 \#6 OR \#10

\#12 \#4 AND \#11

\#13 choose filter "Randomized Controlled Trial"

(3) $\mathrm{CBM}$

(((“blood pressure variability” [Common fields: intelligence]) OR (“fluctuation of blood pressure” [Common fields: intelligence]) OR ("BPV” [Common fields: intelligence]) OR ("blood pressure variability" [Common fields: intelligence])) AND ("randomized" [All: intelligence]) AND (("Chinese medicine" [All: intelligence]) OR ("Chinese patent medicine" [All: intelligence]) OR ("integrated Chinese and western medicine" [All: intelligence]) OR ("granule" [All: intelligence]) OR ("Chinese herbal medicine" [All: intelligence]) OR ("traditional Chinese medicine" [All: intelligence])))

(4) CNKI

(SU = "blood pressure variability" OR SU = "fluctuation of blood pressure" OR SU = "BPV") AND (FT = "traditional Chinese medicine" OR FT = "Chinese patent medicine" OR FT = "integrated Chinese and western medicine" OR FT = "granule" OR FT = "Chinese herbal medicine" OR FT = "Chinese medicine") AND (FT = "randomized")

(5) VIP

(Title or key word $=$ blood pressure variability + fluctuation of blood pressure + BPV) and $($ Any Field $=$ traditional Chinese medicine + integrated Chinese and western medicine + granule + Chinese patent medicine + Chinese herbal medicine + Chinese medicine) and (Any Field $=$ randomized) and (Professional = pharmaceutical and health care) and (Range $=$ all journals)

(6) WanFang database

(title or key word: (blood pressure variability) + title or key word: (fluctuation of blood pressure) + title or key word: (BPV)) * (Chinese medicine + Chinese patent medicine + integrated Chinese and western medicine + granule + Chinese herbal medicine + traditional Chinese medicine) $*$ randomized

out in mainland China. One article [21] was funded by Shandong province science and technology development project.

3.1.2. Population Characteristics. 1103 patients were included, with an average of 85 cases per trial (range from 40 cases to
160 cases). Eight trials [17-21,25-27] included both inpatients and outpatients, three trials [21, 23, 29] only contained outpatients, and two trials $[24,28]$ did not report whether outpatients or inpatients. All trials claimed baseline characteristics were comparable between the groups. Five trials 
PRISMA 2009 Flow Diagram
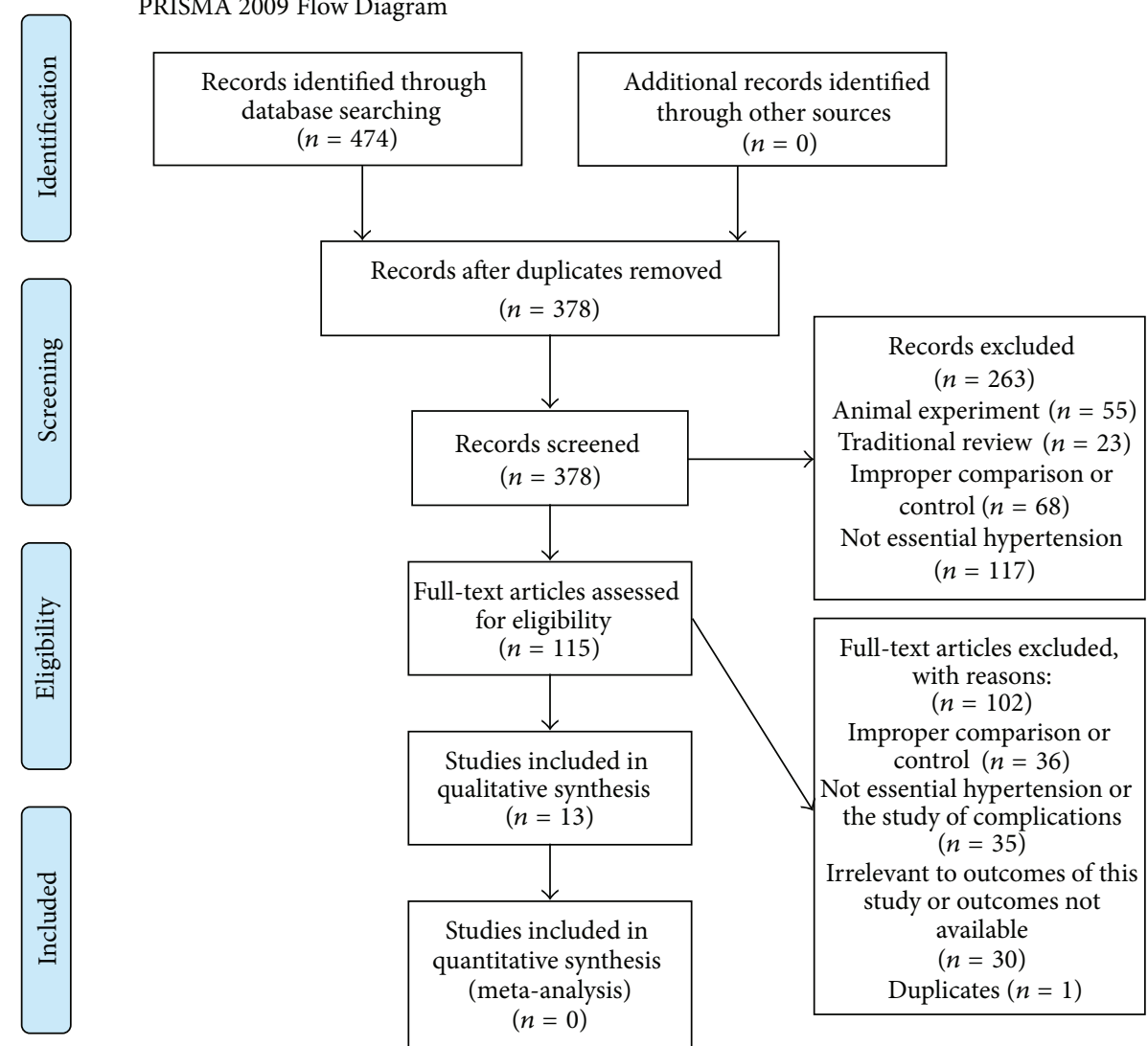

FIGURE 1: Flow chart of study selection.

$[17,23,24,26,29]$ did not report male/female ratio in different groups, three trials $[24,26,28]$ did not report distribution of age, and five [21, 23, 24, 26, 29] papers did not report disease duration between the groups. The remaining trials clearly reported these items. High BP classification was limited to first and second degree, except two trials (one [24] did not report high BP classification and one [25] was not limited classification). One trial [17] explicitly mentioned complications, but there was no significant difference in the distribution and two groups can be comparable. Remaining trials did not report complications.

3.1.3. Comparisons. There were two types of comparisons: (1) Chinese medicine combined with conventional therapy versus conventional therapy alone (twelve trials) and (2) Chinese medicine combined with conventional therapy versus placebo and conventional therapy (one trial). Conventional therapy of six trials [17, 20-22, 26, 29] was amlodipine besylate tablet $5 \mathrm{mg}$ per day. One trial was levamlodipine [28]. One trial was nifedipine controlled released tablet [27]. Two trials $[18,24]$ were CCB combined with ACEI. Two trials were ACEI $[19,23]$. One trial did not report the ingredients of Chinese medicine [25]. There were four dosage forms: decoction, capsule, granule, and tablet. (Herbal medicines and adverse effects in the included trials are presented in Table 4.)
3.1.4. Outcome Measures. Nine trials [17, 19, 22, 24-29] reported adverse events. Two trials [20, 23] clearly reported there was no adverse drug reaction. Two trials [23, 29] reported QOL. No trial reported health-economic indicators or follow-up visit. All trials reported BPV in the form of SD or CV, symptom improvement rate (antihypertensive effect) of which diagnostic criterion was Clinical Research Guideline of New Drugs for Traditional Chinese Medicine. Antihypertensive effect is divided into three levels according to the $B P$ value: markedly effective, effective, and invalid.

3.2. Methodological Quality. Five trials [17, 19, 26, 27, 29] used random number table to generate the random sequence. Two trials $[17,29]$ referred to opaque sealed envelopes. Blinding of participants and personnel was mentioned in only one trial [29] which was double-blinding. No trial blinded the outcome assessors. There was not sufficient information to judge whether outcome assessors were blinded or not. Eleven trials did not miss outcome data, among which two trials [17, 26] clearly claimed no drop-out patient. All trials reported their prespecified primary outcomes except one trial. All trials declared baseline characteristics were comparable. One trial [21] was lack of the inclusion and exclusion criteria. Unfortunately, no trial reported sample size calculation. (Risk of bias summaries are presented in Figure 2.) 


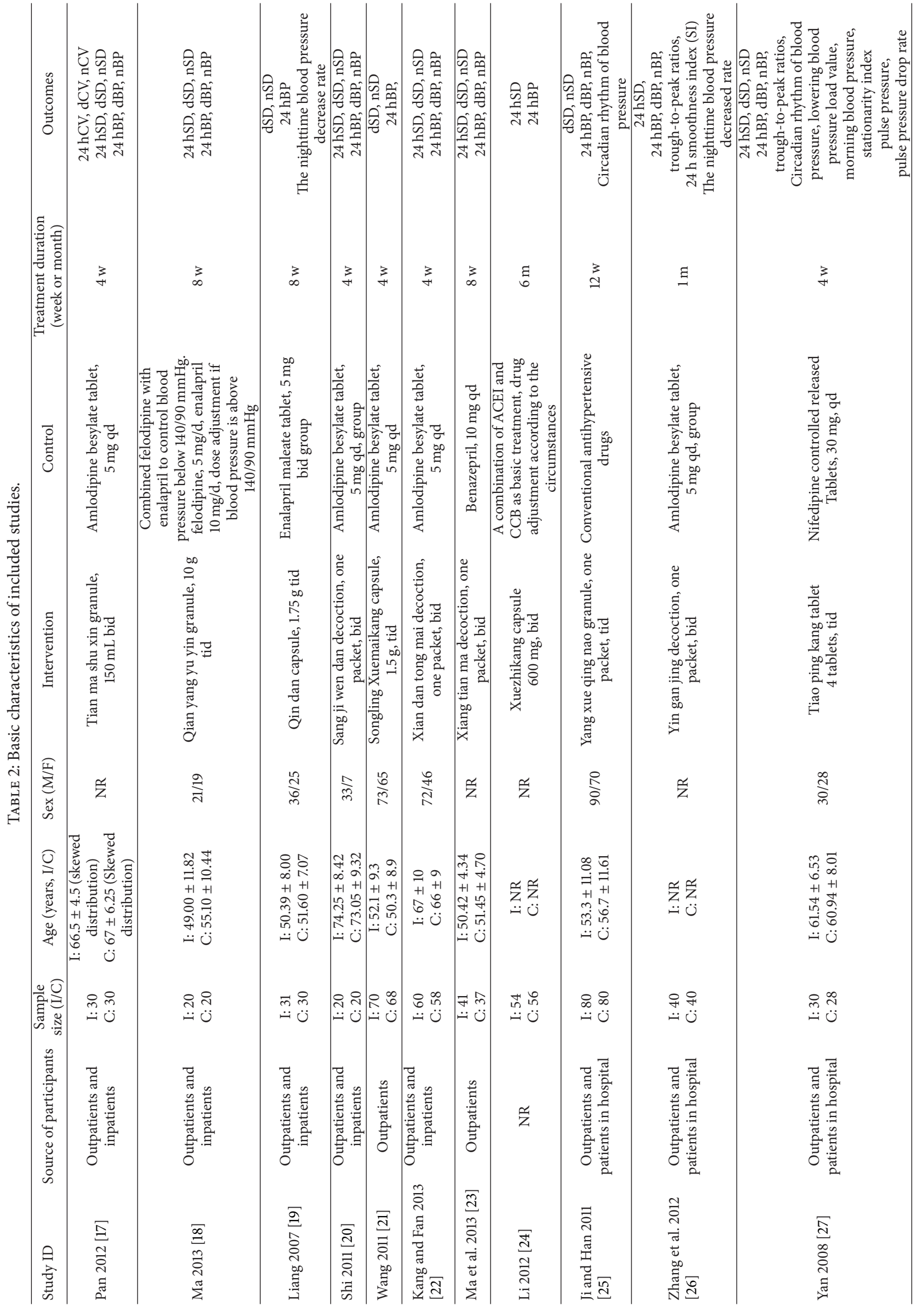




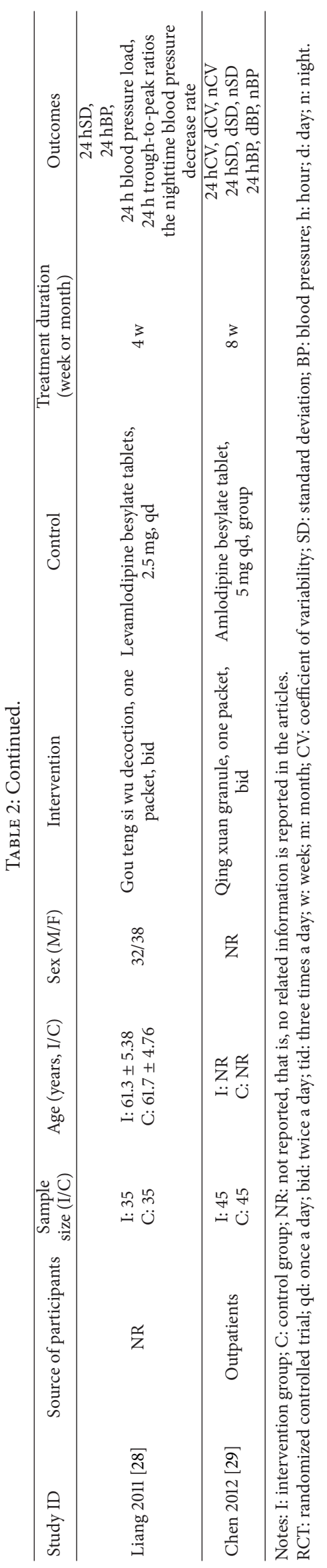




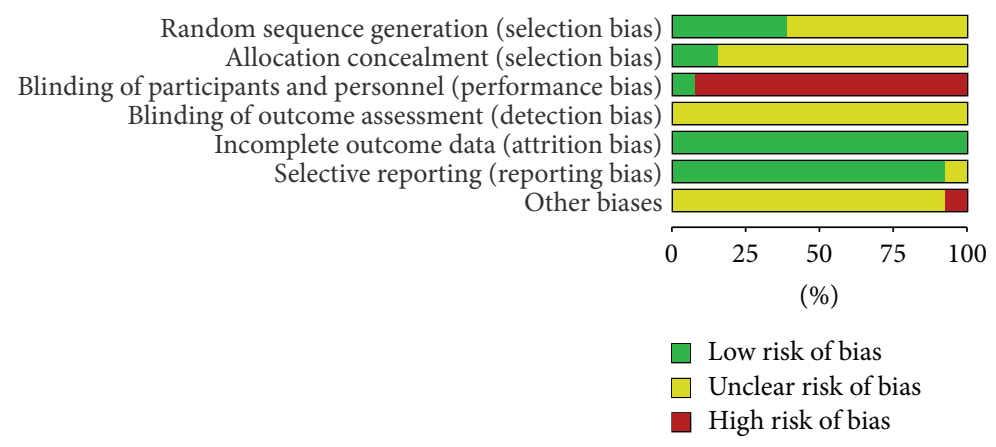

(a)
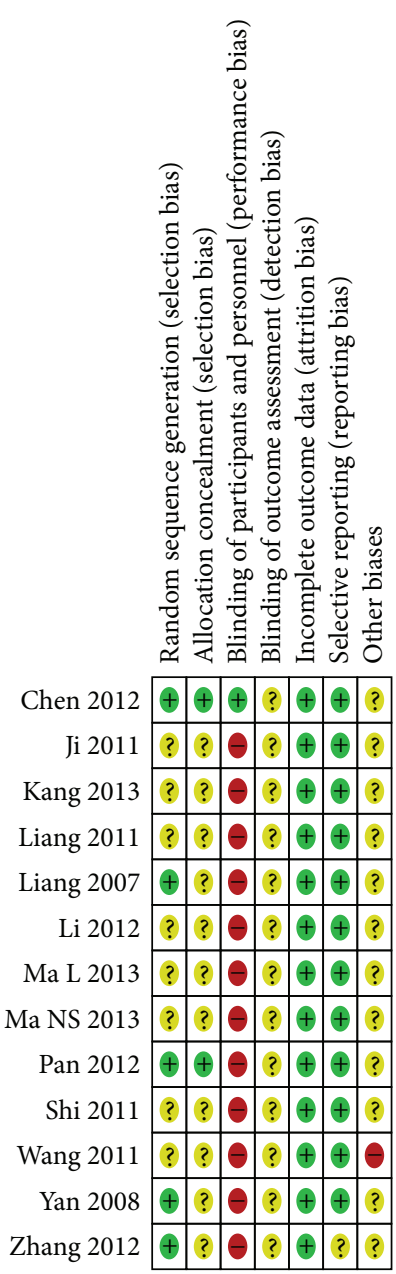

(b)

FiguRE 2: (a) Risk of bias graph: review authors' judgments about each risk of bias item presented as percentages across all included studies. (b) Risk of bias summary: review authors' judgments about each risk of bias item for each included study. “+”: low risk of bias; “?”: unclear risk of bias; or "-" high risk of bias.

3.3. Effects of Interventions. Since every trial had different Chinese herbal medicines as treatment, none of trials could be analyzed by meta-analysis, because of clinical heterogeneity. We presented the effects of interventions by qualitative description, according to the two types of comparisons: Chinese medicine combined with conventional therapy versus the same conventional therapy and Chinese medicine combined with conventional therapy versus placebo combined with the same conventional therapy. All BPV were measured by ambulatory BP meter. BPV mainly was expressed in two forms: SD and CV. (Effect estimate of outcomes is presented in Table 3.)

\subsubsection{Chinese Medicine Combined with Conventional Therapy versus the Same Conventional Therapy}

(1) SD after Treatment. There were nine trials $[17,18,20,22-24$, 26-29] reporting $24 \mathrm{~h}$ systolic SD. Five trials [20, 22-24, 26] found statistical difference between groups. The results have shown that combination therapy is superior to conventional treatment. For traditional Chinese medicine (TCM) combined with amlodipine besylate tablet, three trials showed a reduction of 24 h systolic SD: Sangji Wendan decoction (MD $-2.44 ; 95 \%$ CI -4.38 to $-0.50 ; n=40$ ), Xiandan Tongmai decoction ( $\mathrm{MD}-2.40 ; 95 \% \mathrm{CI}-3.50$ to $-1.30 ; n=118$ ), and Yin gan jing decoction ( $\mathrm{MD}-0.90 ; 95 \% \mathrm{CI}-1.45$ to $-0.35 ; n=80)$. Xiang tian ma decoction combined with benazepril also reduced $24 \mathrm{~h}$ systolic SD (MD -5.20; 95\% CI -6.37 to $-4.03 ; n=78$ ). Xuezhikang capsule was integrated with angiotensin converting enzyme inhibitors (ACEI) and calcium channel blockers (CCB) (MD -1.79; 95\% CI -2.79 to $-0.79 ; n=110)$. There were no significant differences in the other four trials; they were Tianma Shuxin granule combined with amlodipine besylate tablet, Qianyang Yuyin granule combined with felodipine and enalapril, Tiao ping kang tablet combined with nifedipine controlled released tablets, and Gouteng Siwu decoction combined with levamlodipine besylate tablet. 


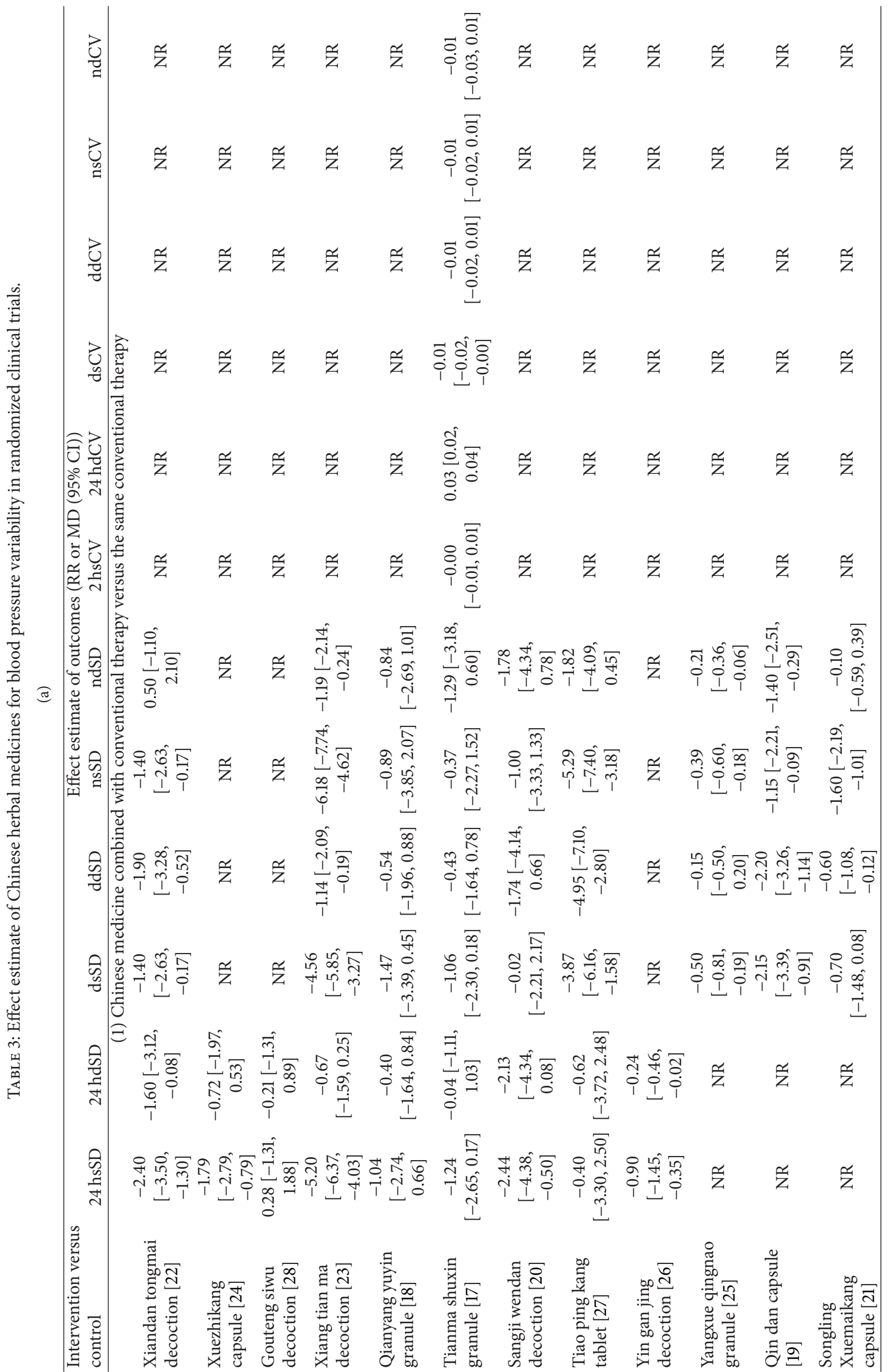




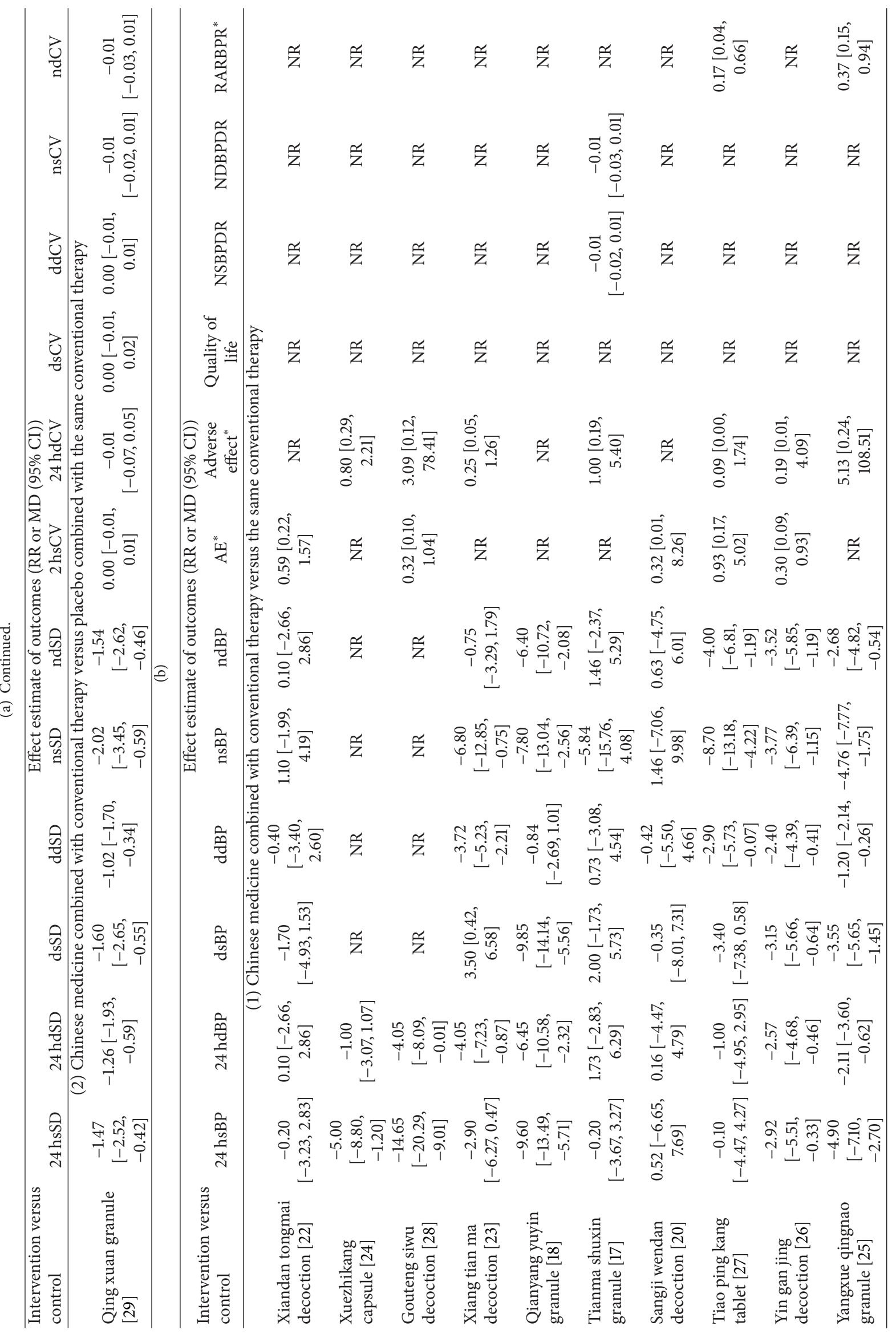




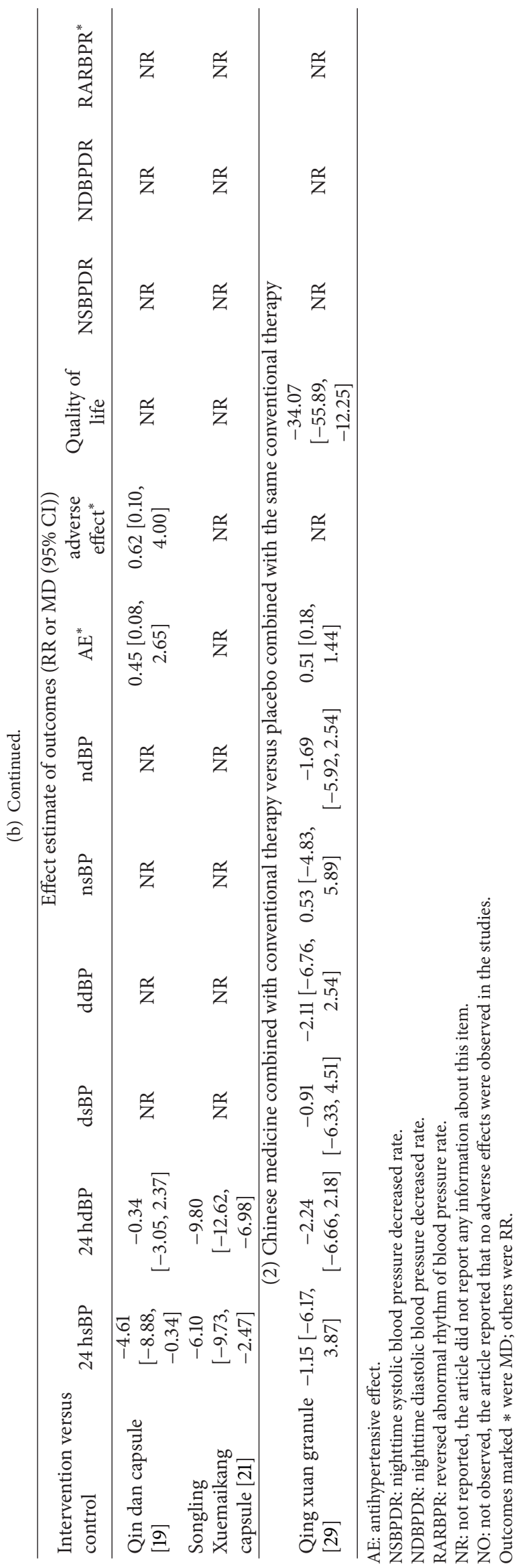




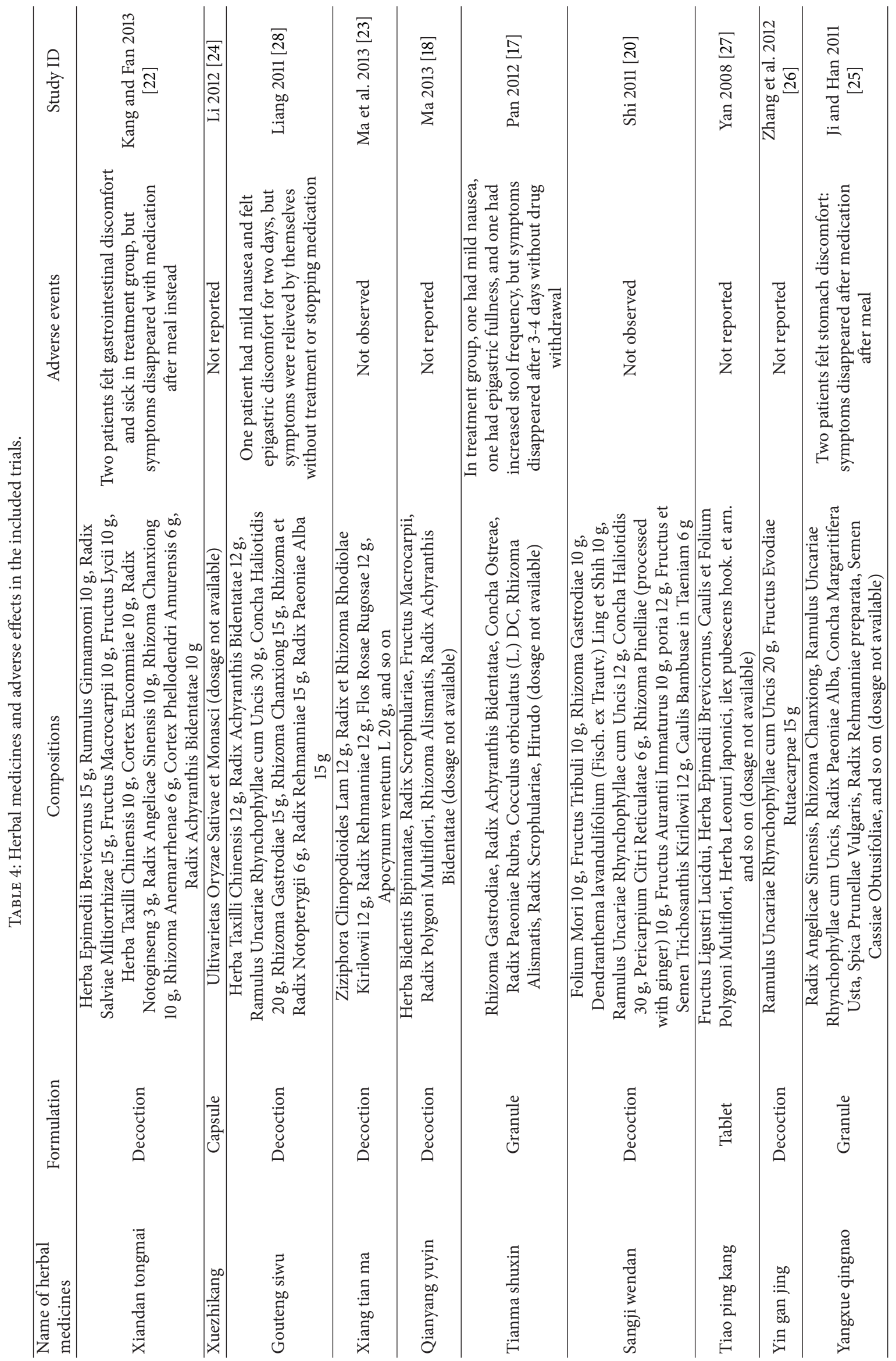




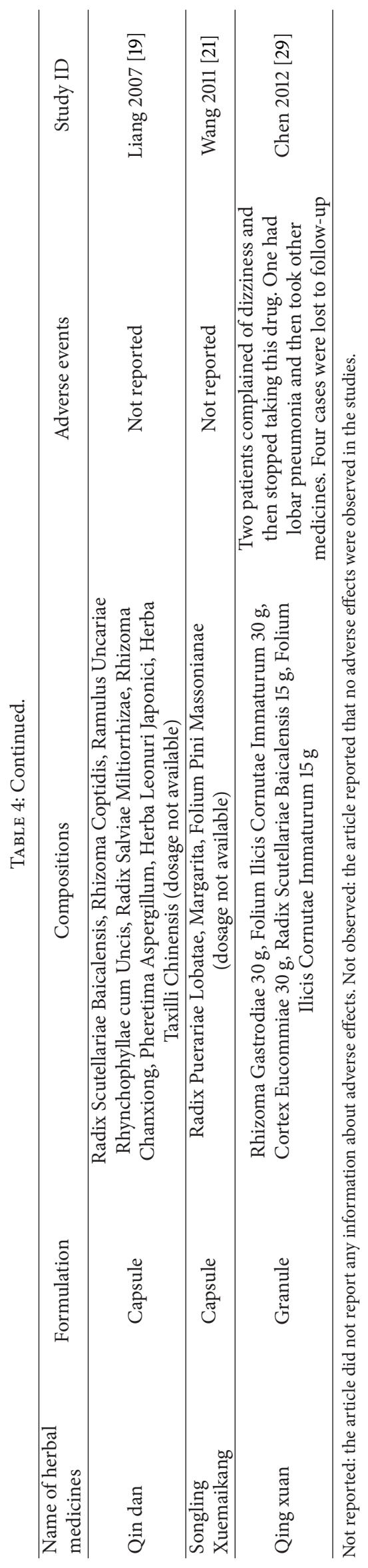


Nine trials $[17,18,20,22-24,26-29]$ reported $24 \mathrm{~h}$ diastolic SD. Two of them which used amlodipine besylate tablet as control group found a significant effect in lowering $24 \mathrm{~h}$ diastolic SD in the experimental group superior to that in the control group, that is Sangji Wendan decoction (MD -2.13 ; $95 \%$ CI -4.34 to $0.08 ; n=40$ ) and Yin gan jing decoction ( $\mathrm{MD}-0.24 ; 95 \% \mathrm{CI}-0.46$ to $-0.02 ; n=80$ ). The other seven trials did not find significant difference between groups: Xiandan Tongmai decoction combined with amlodipine besylate tablet, Tianma Shuxin granule combined with amlodipine besylate tablet, Xiang tian ma decoction combined with benazepril, Xuezhikang capsule integrated with ACEI and CCB, Qianyang Yuyin granule combined with felodipine and enalapril, Tiao ping kang tablet combined with nifedipine controlled released tablets, and Gouteng Siwu decoction combined with levamlodipine besylate tablet.

Day systolic SD was reported by nine trials. Five trials [17$23,25,27]$ significantly lowered this outcome in experimental group superior to that in control group. They were Qin dan capsule plus enalapril maleate tablet ( $\mathrm{MD}-2.15 ; 95 \%$ CI -3.39 to $-0.91 ; n=61$ ), Xiandan Tongmai decoction plus amlodipine besylate tablet (MD $-1.40 ; 95 \% \mathrm{CI}-2.63$ to $-0.17 ; n=118)$, Xiang tian ma decoction plus benazepril (MD -4.56; 95\% CI $-5.85,-3.27 ; n=78)$, Yangxue Qingnao granule plus conventional antihypertensive drugs (MD $-0.50 ; 95 \%$ CI -0.81 to $-0.19 ; n=160$ ), and Tiao ping kang tablet plus nifedipine controlled released tablets (MD -3.87 ; $95 \%$ CI -6.16 to $-1.58 ; n=58$ ). The other four trials did not find significant difference between groups, one trial about Qianyang Yuyin granule plus felodipine with enalapril and three trials compared amlodipine besylate tablets, that were Tianma Shuxin granule, Sangji Wendan decoction, and Songling Xuemaikang capsule.

Day diastolic SD was reported by nine trials [17-23, 2527]. Five trials found the effect of lowering this outcome in experimental group superior to that in control group. They were Qin dan capsule combined with enalapril maleate tablet (MD $-2.20 ; 95 \% \mathrm{CI}-3.26$ to $-1.14 ; n=61$ ), Songling Xuemaikang capsule combined with amlodipine besylate tablet ( $\mathrm{MD}-0.60$; $95 \% \mathrm{CI}-1.08$ to $-0.12 ; n=138$ ), Xiandan Tongmai decoction combined with amlodipine besylate tablet (MD $-1.90 ; 95 \% \mathrm{CI}-3.28$ to $-0.52 ; n=118)$, Xiang tian ma decoction combined with benazepril (MD -1.14; 95\% CI -2.09 to $-0.19 ; n=78$ ), and Tiao ping kang tablet combined with nifedipine controlled released Tablets $(\mathrm{MD}-4.95 ; 95 \% \mathrm{CI}-7.10$ to $-2.80 ; n=58)$. Although the other four trials had reported this outcome too, there was no statistically significant difference. The four trials were Tianma Shuxin granule combined with amlodipine besylate tablet, Qianyang Yuyin granule combined with felodipine and enalapril, Yangxue Qingnao granule combined with conventional antihypertensive drugs, and Sangji Wendan decoction combined with amlodipine besylate tablet.

Nine trials [17-23, 25, 27] reported night systolic SD. Six trials showed a reduction of night systolic SD. Two of them are compared with amlodipine besylate tablet; they were Songling Xuemaikang capsule (MD -0.60; 95\% CI - 1.08 to $-0.12 ; n=138$ ) and Xiandan Tongmai decoction (MD $-1.90 ; 95 \%$ CI -3.28 to $-0.52 ; n=118$ ), besides, Xiang tian ma decoction combined with benazepril (MD -1.14; 95\% CI -2.09 to $-0.19 ; n=78)$, Qin dan capsule combined with enalapril maleate tablet (MD -2.20 ; $95 \% \mathrm{CI}-3.26$ to -1.14 ; $n=61$ ), Tiao ping kang tablet combined with nifedipine controlled released tablets $(\mathrm{MD}-4.95 ; 95 \% \mathrm{CI}-7.10$ to -2.80 ; $n=58$ ), and Yangxue Qingnao granule combined with conventional antihypertensive drugs (MD -0.39 ; 95\% CI -0.60 to $-0.18 ; n=160)$. Three trials showed no significant difference, that is, Tianma Shuxin granule combined with amlodipine besylate tablet, Qianyang Yuyin granule combined with felodipine and enalapril, and Sangji Wendan decoction combined with amlodipine besylate tablet.

There were nine trials [17-23, 25, 27] reporting night diastolic SD. Three trials showed significant reduction of this outcome: Xiang tian ma decoction combined with benazepril (MD $-1.19 ; 95 \% \mathrm{CI}-2.14$ to $-0.24 ; n=78$ ), Yangxue Qingnao granule combined with conventional antihypertensive drugs (MD $-0.21 ; 95 \% \mathrm{CI}-0.36$ to $-0.06 ; n=160$ ), and Qin dan capsule combined with enalapril maleate tablet (MD -1.40; $95 \%$ CI -2.51 to $-0.29 ; n=61)$. Results of the remaining six trials had no statistical differences.

(2) CV after Treatment. Only one trial (Tianma Shuxin granule combined with amlodipine besylate tablet) reported CV. There were statistically significant differences in two outcomes. There was a reduction in day systolic CV in experimental group superior to that in control group (MD -0.01 ; $95 \% \mathrm{CI}-0.02$ to $-0.00 ; n=60)$. There was a reduction in $24 \mathrm{~h}$ diastolic CV in experimental group inferior to that in control group (MD 0.03; 95\% CI 0.02 to $0.04 ; n=60$ ). Nevertheless, there was a reduction in day systolic $\mathrm{CV}$ in control group inferior to that in experimental group (MD -0.01 ; 95\% CI -0.02 to $-0.00 ; n=60)$. There was no statistically significant advantage in reducing $\mathrm{CV}$ in experimental group in terms of 24 h systolic CV, day diastolic CV, night systolic CV and night diastolic CV.

(3) BP after Treatment. Seven trials [18, 19, 21, 24-26, 28] presented that integrative medicine had the advantage in reducing $24 \mathrm{~h}$ systolic BP. Six trials [18, 21, 23, 25, 26, 28] presented that integrative medicine had the advantage in reducing $24 \mathrm{~h}$ diastolic BP. There were three trials [18, 25, 26] showing that integrative medicine had the advantage in reducing day systolic BP; however, one trial [23] had the opposite result. Four trials [23, 25-27] presented that integrative medicine had the advantage in reducing day diastolic BP. There were five trials [18, 23, 25-27] showing that integrative medicine had the advantage in reducing night systolic BP. Four trials [18, 25-27] presented that integrative medicine had the advantage in reducing night diastolic BP.

(4) Antihypertensive Effect. Antihypertensive effect was measured taking clinical guideline of new drugs for TCM as standard. Only one trial [26] showed treatment group superior to control group in improving antihypertensive effect.

(5) QOL. One trial [23] referred to QOL but did not report which specific test scale it used.

(6) Nighttime BP Decreased Rate. Three trials [19, 26, 28] reported this outcome. Only one trial [26] presented that 
combination therapy is superior to conventional treatment in nighttime BP decreased rate of both systolic BP and diastolic BP.

(7) Frequency of Reversed Abnormal Rhythm of BP. Two trials $[25,27]$ showed treatment group superior to control group in increasing frequency of reversed abnormal rhythm of BP.

\subsubsection{Chinese Medicine Combined with Conventional Therapy} versus Placebo Combined with the Same Conventional Therapy. There was only one trial [29] under this category, that is, Qing xuan granule combined with amlodipine besylate tablet versus placebo combined with amlodipine besylate tablet.

(1) SD after Treatment. All the outcomes about SD of this trial were of significant statistical heterogeneity. Qing xuan granule combined with amlodipine besylate tablet had a better efficacy than the control in terms of lowering $24 \mathrm{~h}$ systolic SD (MD $-1.47 ; 95 \% \mathrm{CI}-2.52$ to $-0.42 ; n=90), 24 \mathrm{~h}$ diastolic SD (MD $-1.26 ; 95 \% \mathrm{CI}-1.93$ to $-0.59 ; n=90)$, day systolic SD (MD $-1.60 ; 95 \% \mathrm{CI}-2.65$ to $-0.55 ; n=90)$, day diastolic SD (MD $-1.02 ; 95 \%$ CI -1.70 to $-0.34 ; n=90)$, night systolic SD (MD $-2.02 ; 95 \% \mathrm{CI}-3.45$ to $-0.59 ; n=90)$, and night diastolic SD (MD $-1.54 ; 95 \% \mathrm{CI}-2.62$ to $-0.46 ; n=90)$.

(2) CV after Treatment. No noteworthy statistical differences of this endpoint in any of the trials were noted.

(3) BP after Treatment. There was no significant difference between the experimental and the control groups regarding this outcome which contained $24 \mathrm{~h}$ systolic BP, $24 \mathrm{~h}$ diastolic $\mathrm{BP}$, day systolic BP, day diastolic BP, night systolic BP, and night diastolic BP.

(4) Antihypertensive Effect. There was no significant difference between the experimental and the control groups regarding this outcome.

(5) QOL. There was also no significant difference between the experimental and the control groups regarding this outcome which was measured by SF-36 health related QOL scale.

(6) Nighttime BP Decreased Rate. It was not reported in this trial.

(7) Frequency of Reversed Abnormal Rhythm of BP. It was not reported in this trial.

3.4. Safety. There were 9 trials reporting 50 cases of adverse events. The experimental groups had 18 patients with side reactions, and the control groups had 29 patients with side reactions. Three patients had adverse events but with no specific group information. No significant difference about adverse events was found between two groups. The most commonly reported adverse events in the 9 trials were intestinal disturbance (abdominal distension, nausea, and constipation) $[17,22,25,28]$, ankle edema [17, 27], dizziness [22, 27, 29], palpitation [22], facial flushing [26], and dry cough $[19,24]$.

\section{Discussion}

There are thirteen RCTs including 1103 participants that were included in this systematic review. From this review, we may be able to speculate that both the TCM and combination therapy have a significant effect in lowering BP, lowering BPV, and adjusting the circadian rhythm. As we known, efficacy of amlodipine besylate tablets in lowering BP variation is better than other western medicines. Most of trials in this paper selected amlodipine besylate tablets as conventional therapy in the compare group of Chinese medicine combined with conventional therapy versus the same conventional therapy. One trial took placebo combined with conventional therapy as control group. According to TCM theories, the treatment is based on syndrome differentiation, and even the same disease could have a variety of syndromes; therefore, the treatments could be modified with different Chinese herbal medicines. Although the differences in TCM prescription, forms, course of treatment, and control drugs prevent us from performing meta-analysis which could provide precise effect estimate of intervention, from Table 4 we gladly found that there were two kinds of TCM (Ramulus Uncariae Rhynchophyllae cum Uncis and Radix Achyranthis Bidentatae) that appeared most. The most TCM syndromes were yin deficiency and yang excess. This is also consistent with the theory of TCM. It was confirmed that the integrative medicine based on syndrome differentiation, a person-centered and balanced medicine [30], was safer and more effective than western medicine alone in the treatment of hypertension [31]. Adverse reaction related to Chinese medicine to reduce $\mathrm{BPV}$ is relatively rare. Only a few patients had ankle edema, dizziness, and nausea during the treatment.

It is too early to recommend this conclusion to clinical practitioners considering the limitations of this review. Firstly, the methodological quality of included trials in the review generally needs to be improved. Studies have shown that the degree of rigorous design and quality of methodology of the study have a direct impact on the effectiveness of intervention $[32,33]$. The randomization was unclear in most of trials. Only five RCTs described random allocation in detail, and two RCTs mentioned random hidden method. Blinding is an effective way to control measurement bias; however, only one trial mentioned double-blind; no trial reported estimation of sample size. Moreover, this review focuses on short-term BPV and all of the included trials did not have follow-up research, so we cannot evaluate longterm effect of Chinese herbal medicine for BPV. In addition, all the trials did not publish their protocols, so we can only judge their reporting bias by a compromise method, which is comparing whether the outcomes mentioned in method and results are consistent. Two trials reported QOL, but only one trial evaluated QOL by certain scale. Fifth, due to the clinical features of hypertension, hypertensive patients commonly also suffered from other diseases, such as coronary heart disease or hyperlipidemia, but only a small number of clinical trials on the baseline data reported these accompanied diseases. Most of the other tests did not report this information, but we cannot rule out the possibility of patients with accompanied disease. Sixth, we only included 
13 trials with a relative small sample size in this review, and we failed to perform meta-analysis. Therefore, further rigorously designed RCTs are needed before recommending TCM to patients with hypertension. Moreover, we suggest that the design and reporting of RCTs on TCM strictly comply with both CONSORT statement [34] and that for herbal interventions [35].

\section{Conclusion}

TCM showed potentially short-term beneficial effects on BPV. However, because of small sample size and potential bias of most trials, this result should be interpreted with caution. More high quality trials, safety evidence, and longterm effects are warranted before TCM is recommended as an alternative or complementary medicine for BPV in hypertension patients. Information of QOL and economic effectiveness should also be paid more attention in future clinical trials.

\section{Conflict of Interests}

The authors declare that there is no conflict of interests regarding the publication of this paper.

\section{Authors' Contribution}

Hao $\mathrm{Xu}$ conceived, designed, and revised this systematic review; Jianping Liu designed and revised this systematic review and provided methodological guidance; Zhuo Chen developed the search strategy, did the literature selection, data extraction, and analysis, and wrote the paper; Liqiong Wang helped to develop the search strategy, did literature selection and data extraction, and offered many suggestions; Guoyan Yang assisted to correct mistakes in the paper and checked interpretation.

\section{References}

[1] P. M. Rothwell, S. C. Howard, E. Dolan et al., "Prognostic significance of visit-to-visit variability, maximum systolic blood pressure, and episodic hypertension," The Lancet, vol. 375, no. 9718, pp. 895-905, 2010.

[2] C. Tsioufis, I. Andrikou, C. Thomopoulos, D. Petras, A. Manolis, and C. Stefanadis, "Comparative prognostic role of nighttime blood pressure and nondipping profile on renal outcomes," American Journal of Nephrology, vol. 33, no. 3, pp. 277-288, 2011.

[3] G. Parati, X. Liu, and J. E. Ochoa, "Clinical relevance of visitto-visit blood pressure variability: impact on renal outcomes," Journal of Human Hypertension, vol. 28, pp. 403-409, 2014.

[4] P. Muntner, D. Shimbo, M. Tonelli, K. Reynolds, D. K. Arnett, and S. Oparil, "The relationship between visit-to-visit variability in systolic blood pressure and all-cause mortality in the general population: findings from NHANES III, 1988 to 1994," Hypertension, vol. 57, no. 2, pp. 160-166, 2011.

[5] G. Mancia, G. Parati, M. Hennig et al., "Relation between blood pressure variability and carotid artery damage in hypertension: Baseline data from the European Lacidipine Study on Atherosclerosis (ELSA)," Journal of Hypertension, vol. 19, no. 11, pp. 1981-1989, 2001.
[6] A. J. Webb, U. Fischer, Z. Mehta, and P. M. Rothwell, "Effects of antihypertensive-drug class on interindividual variation in blood pressure and risk of stroke: a systematic review and metaanalysis," The Lancet, vol. 375, no. 9718, pp. 906-915, 2010.

[7] ALLHAT Officers and Coordinators for the ALLHAT Collaborative Research Group, "The Antihypertensive and LipidLowering Treatment to Prevent Heart Attack Trial. Major outcomes in high-risk hypertensive patients randomized to angiotensin-converting enzyme inhibitor or calcium channel blocker vs diuretic: The Antihypertensive and Lipid-Lowering Treatment to Prevent Heart Attack Trial (ALLHAT)," The Journal of the American Medical Association, vol. 288, no. 23, pp. 2981-2997, 2002.

[8] H.-H. Xie, F.-M. Shen, L.-P. Xu, P. Han, C.-Y. Miao, and D.-F. $\mathrm{Su}$, "Reduction of blood pressure variability by combination therapy in spontaneously hypertensive rats," Journal of Hypertension, vol. 25, no. 11, pp. 2334-2344, 2007.

[9] R. Sega, G. Corrao, M. Bombelli et al., "Blood pressure variability and organ damage in a general population: results from the PAMELA study," Hypertension, vol. 39, no. 2, pp. 710-714, 2002.

[10] Z. Y. Zhao, Z. Y. Zhao, Y. Q. Wang, Z. H. Yan, J. Cui, and Y. Y. Li, "Quantitative study of circadian variations of ambulatory blood pressure in Chinese healthy, hypertensive, and diabetes subjects," Clinical and Experimental Hypertension, vol. 27, no. 2-3, pp. 187-194, 2005.

[11] Y. Liu, J. C. Zhang, D. Z. Shi et al., "Fluctuant hypertension and platelet activation: current situations and control strategies with integrative medicine," Zhongguo Zhong Xi Yi Jie He Za Zhi, vol. 33, no. 7, pp. 869-872, 2013.

[12] World Health Organization and International Society of Hypertension Writing Group, "2003 World Health Organization (WHO)/International Society of Hypertension (ISH) statement on management of hypertension," Clinical and Experimental Hypertension, vol. 21, no. 5-6, pp. 1009-1060, 1999.

[13] World Health Organization and International Society of Hypertension Writing Group, "2003 World Health Organization (WHO)/International Society of Hypertension (ISH) statement on management of hypertension," Journal of Hypertension, vol. 21, no. 11, pp. 1983-1992, 2003.

[14] Chinese Hypertension Prevention Guide Revision Committee, Chinese Hypertension Prevention Guide (2005 Revision), People's Health Publishing House, Beijing, China, 2006.

[15] Chinese Hypertension Prevention Guide Revision Committee, "Chinese hypertension prevention guide 2010," Chinese Jounal of Hypertension, vol. 19, no. 8, pp. 701-708, 2011.

[16] J. P. T. Higgins and S. Green, Cochrane Handbook for Systematic Reviews of Interventions. Version 5.1.0, The Cochrane Collaboration, Oxford, UK, 2011, http://handbook.cochrane.org/.

[17] L. M. Pan, The Study of TCM Syndromes in Elderly Hypertensive Patients and Evaluation between Syndromes and Blood Pressure Variability and Treatment with TCM, China Academy of Chinese Medical Sciences, Beijing, China, 2012.

[18] N. S. Ma, Study on Influence of Qian Yang Yu Yin Granules on Blood Pressure Variability of the Patients with Hypertension, Nanjing University of Chinese Medicine, Nanjing, China, 2013.

[19] L. Liang, Clinic Study on the Effects of Qin Dan Capsule in Treating Essential Hypertension Patients of "Liver Fire and Blood Stagnation", Shandong University, Jinan, China, 2007.

[20] M. Shi, The Clinical Study of Sang Ji Wen Dan Decoction Treatment on Hypertension in Old Patients with Liver Yang Hyper Activity and Phlegm-Fire Syndrome, Nanjing University of Chinese Medicine, Nanjing, China, 2011. 
[21] F. Q. Wang, "Efficacy observation of Song ling xue mai kang combined with amlodipine in treating essential hypertension patients," Jilin Journal of Traditional Chinese Medicine, vol. 31, no. 2, pp. 149-150, 2011.

[22] X. X. Kang and P. Fan, "The treatment of Xian Dan Tong Mai decoction in elderly hypertension patients," Shandong Journal of Traditional Chinese Medcine, vol. 32, no. 10, pp. 723-725, 2013.

[23] L. Ma, J. He, and H. B. Li, "The impact of Xiang Tian Ma decoction on blood pressure variability in patients of deficiency of Yin and internal dry," Xinjiang Journal of Traditional Chinese Medicine, vol. 31, no. 1, pp. 11-13, 2013.

[24] Y. L. Li, "Effects of combined application of xuezhikang capsule with hypotensive drug on ambulatory blood pressure and blood pressure Variability," Guide of China Medicine, vol. 10, no. 6, pp. 41-43, 2012.

[25] R. L. Ji and Q. H. Han, "Efficacy research on Yang xue qing nao particles in patients with hypertension," Chinese Journal of Integrative Medicine on Cardio-cerebrovascular Disease, vol. 9, no. 3, pp. 263-265, 2011.

[26] W. N. Zhang, H. F. Huang, and X. Z. Tang, "Influence of medicine leading to the liver channel in antihypertensive efficacy of amlodipine in the elderly hypertensive patients," in The Tenth TCM Academic Communication of Prevention and Treatment of Senile Diseases, Xianmen, China, 2012.

[27] Z. Q. Yan, The Effect of Tiao Ping Kang Discus in Diurnal Rhythm and the Clinical Therapeutic Effect in Essential Hypertension Patients, Guangzhou University of Chinese Medicine, Guangzhou, China, 2008.

[28] C. G. Liang, The Study on the "Gou Teng Si Wu Tang" Treating Level 1,2 Elderly Hypertension, Shandong University of Chinese Medicine, Jinan, China, 2011.

[29] J. Chen, The Intervention Effects of Qingxuan Granule on Endothelial Injury Induced by Inflammatory Reaction of Hypertensive Patients, China Academy of Chinese Medical Sciences, Beijing, China, 2012.

[30] X.-G. Sun, W.-K. Wu, and Z.-P. Lu, "Chinese integrative medicine: translation toward person-centered and balanced medicine," Chinese Journal of Integrative Medicine, vol. 18, no. 1, pp. 3-6, 2012.

[31] S.-L. Chen, X.-Y. Liu, W.-M. Xu, W.-Y. Mei, and X.-L. Chen, "Clinical study of Western medicine combined with Chinese medicine based on syndrome differentiation in the patients with polarized hypertension," Chinese Journal of Integrative Medicine, vol. 18, no. 10, pp. 746-751, 2012.

[32] N. Freemantle, M. Calvert, J. Wood, J. Eastaugh, and C. Griffin, "Composite outcomes in randomized trials: greater precision but with greater uncertainty?" The Journal of the American Medical Association, vol. 289, no. 19, pp. 2554-2559, 2003.

[33] L. L. Kjaergard, J. Villumsen, and C. Gluud, "Reported methodologic quality and discrepancies between large and small randomized trials in meta-analyses," Annals of Internal Medicine, vol. 135, no. 11, pp. 982-989, 2001.

[34] K. F. Schulz, D. G. Altman, and D. Moher, "CONSORT 2010 statement: updated guidelines for reporting parallel group randomized trials," Annals of Internal Medicine, vol. 152, no. 11, pp. 726-732, 2010.

[35] J. J. Gagnier, H. Boon, P. Rochon, D. Moher, J. Barnes, and C. Bombardier, "Reporting randomized, controlled trials of herbal interventions: an elaborated CONSORT statement," Annals of Internal Medicine, vol. 144, no. 5, pp. 364-367, 2006. 


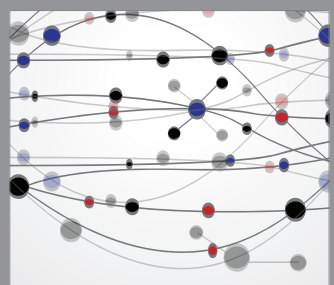

The Scientific World Journal
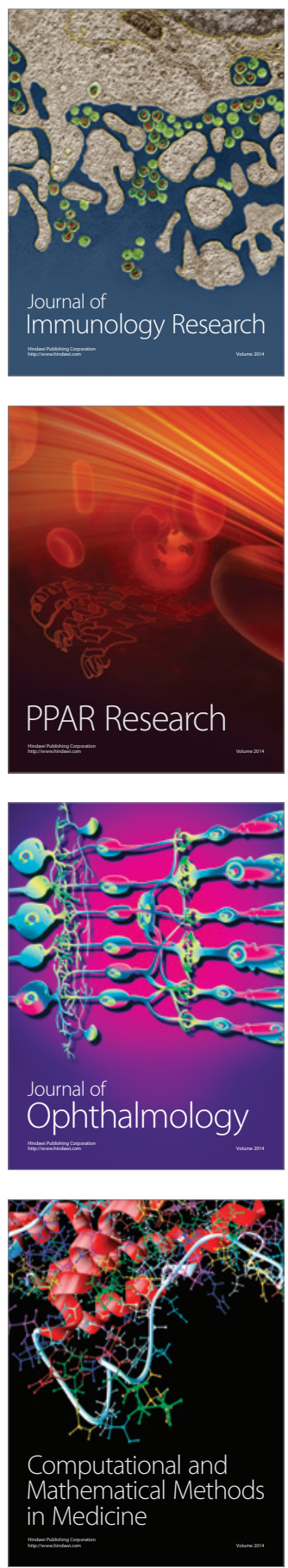

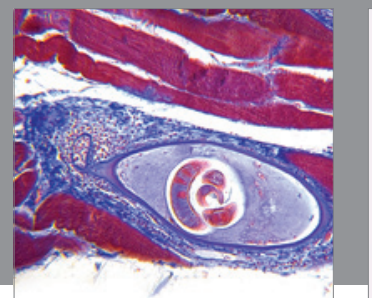

Gastroenterology

Research and Practice
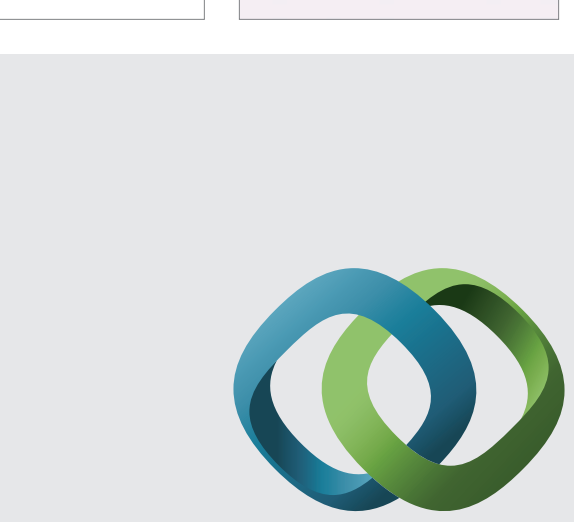

\section{Hindawi}

Submit your manuscripts at

http://www.hindawi.com
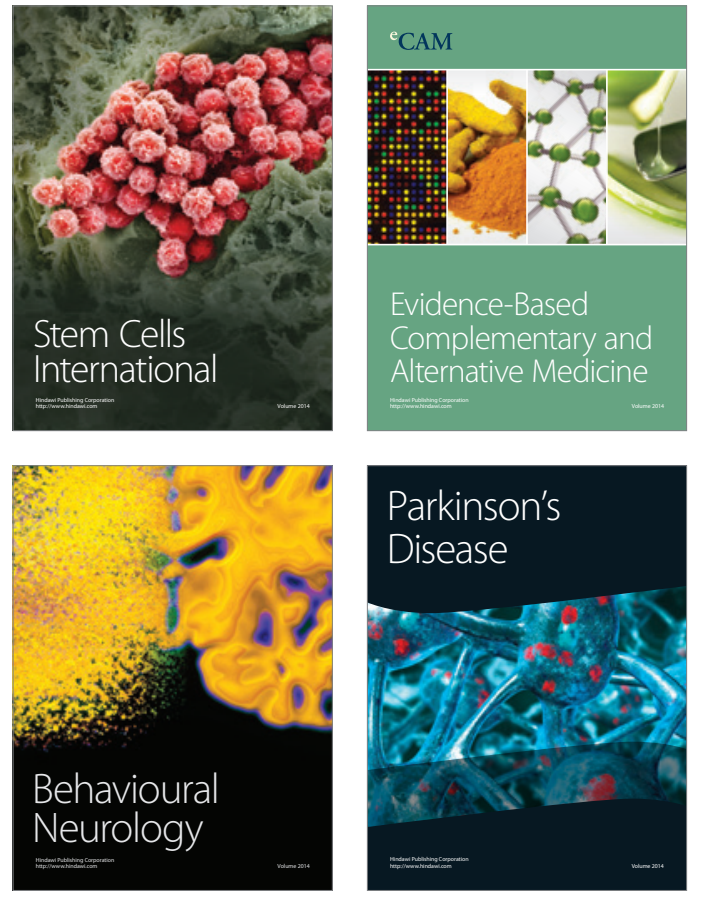
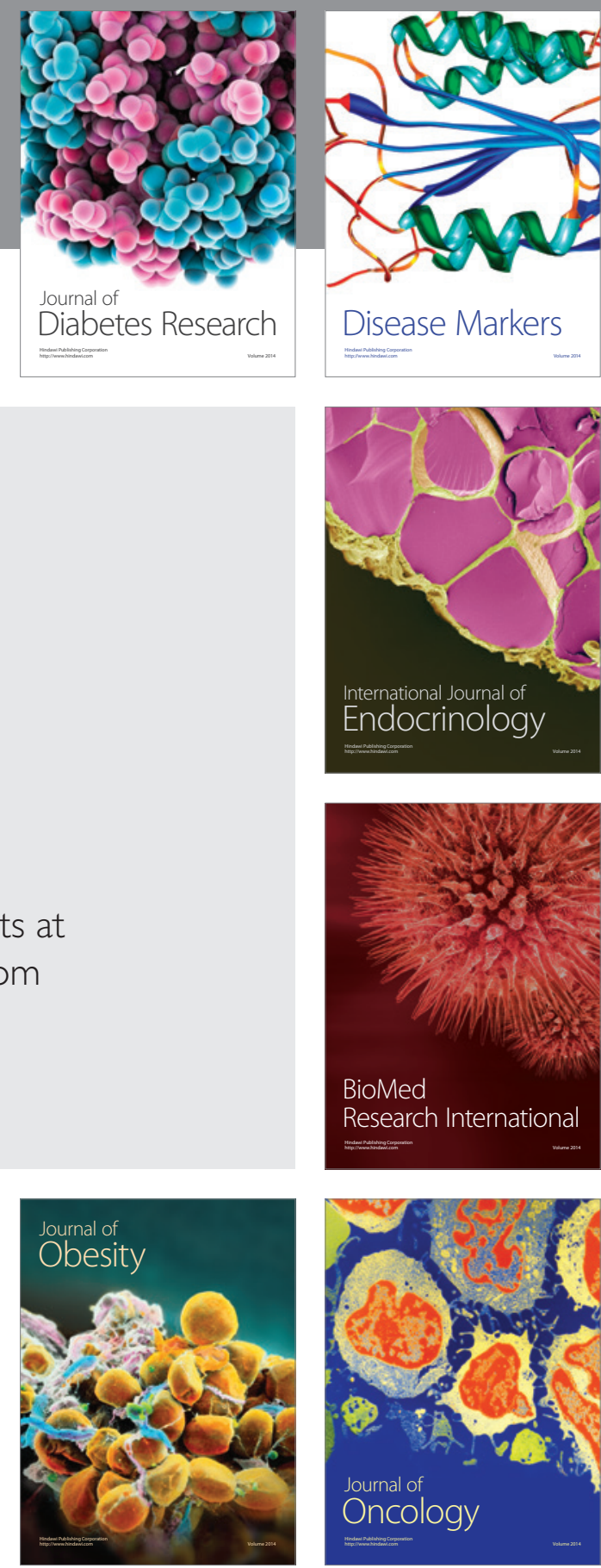

Disease Markers
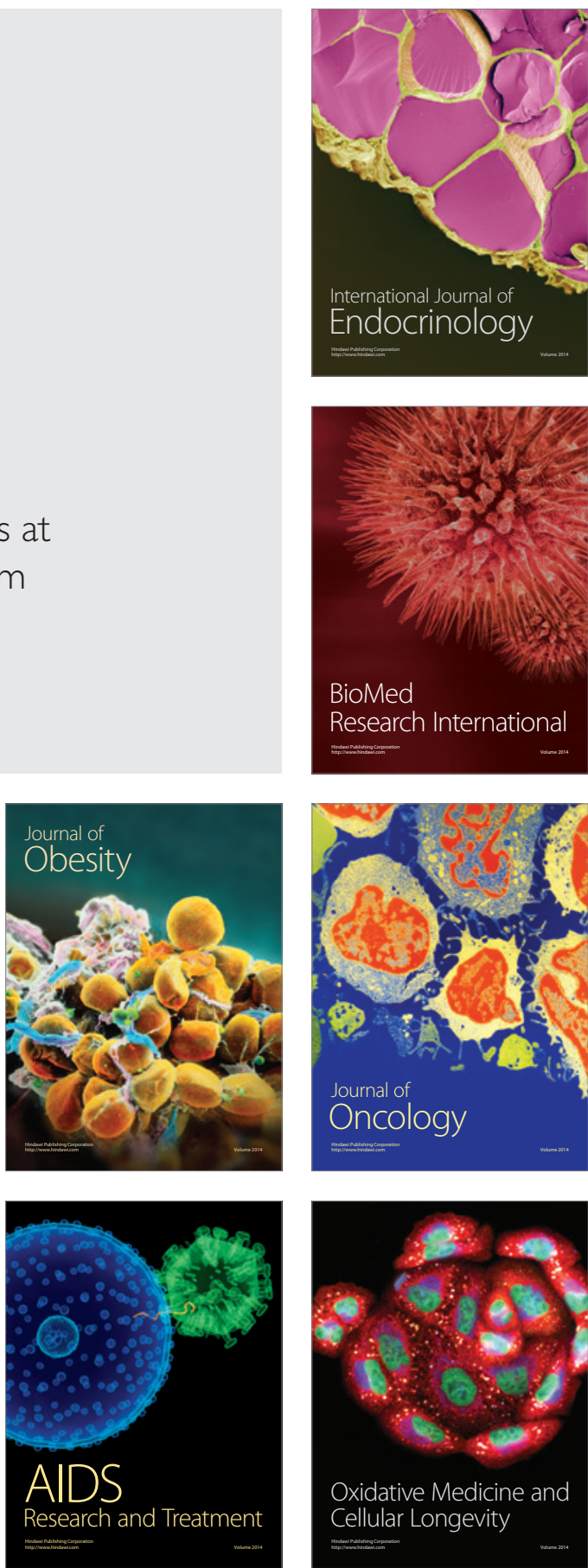